\title{
Intestinal Inflammation Induced by Soybean Meal Ingestion Increases Intestinal Permeability and Neutrophil Turnover Independently of Microbiota in Zebrafish
}

OPEN ACCESS

Edited by:

Geert Wiegertjes,

Wageningen University and

Research, Netherlands

Reviewed by:

Sylvia Brugman,

Wageningen University and

Research, Netherlands

Bjørn Erik Vind Koch,

Leiden University, Netherlands

*Correspondence:

Carmen G. Feijoo

cfeijoo@unab.c

Specialty section:

This article was submitted to Comparative Immunology,

a section of the journal

Frontiers in Immunology

Received: 23 December 2019 Accepted: 26 May 2020 Published: 24 July 2020

Citation:

Solis CJ, Hamilton MK, Caruffo M,

Garcia-Lopez JP, Navarrete P, Guillemin K and Feijoo CG (2020) Intestinal Inflammation Induced by

Soybean Meal Ingestion Increases Intestinal Permeability and Neutrophil

Turnover Independently of Microbiota in Zebrafish. Front. Immunol. 11:1330.

doi: 10.3389/fimmu.2020.01330

\section{Camila J. Solis ${ }^{1,2}$, M. Kristina Hamilton ${ }^{3}$, Mario Caruffo ${ }^{1,4}$, Juan P. Garcia-Lopez ${ }^{1}$, Paola Navarrete ${ }^{2,5}$, Karen Guillemin ${ }^{3,6}$ and Carmen G. Feijoo ${ }^{1,2 *}$}

${ }^{1}$ Fish Immunology Laboratory, Faculty of Life Sciences, Universidad Andres Bello, Santiago, Chile, ${ }^{2}$ Millennium Nucleus in the Biology of Intestinal Microbiota, Santiago, Chile, ${ }^{3}$ Institute of Molecular Biology, University of Oregon, Eugene, OR, United States, ${ }^{4}$ Escuela de Biotecnología, Facultad de Ciencias, Universidad Santo Tomás, Santiago, Chile, ${ }^{5}$ Laboratory of Microbiology and Probiotics, Institute of Nutrition and Food Technology (INTA), University of Chile, Santiago, Chile, ${ }^{6}$ Humans and the Microbiome Program, Canadian Institute for Advanced Research, Toronto, ON, Canada

Intestinal inflammation is a condition shared by several intestinal chronic diseases, such as Crohn's disease and ulcerative colitis, with severely detrimental consequences in the long run. Current mammalian models have considerably increased understanding of this pathological condition, highlighting the fact that, in most of the cases, it is a highly complex and multifactorial problem and difficult to deal with. Thus, there is an increasingly evident need for alternative animal models that could offer complementary approaches that have not been exploited in rodents, thereby contributing to a different view on the disease. Here, we report the effects of a soybean meal-induced intestinal inflammation model on intestinal integrity and function as well as on neutrophil recruitment and microbiota composition in zebrafish. We find that the induced intestinal inflammation process is accompanied by an increase in epithelial permeability in addition to changes in the mRNA levels of different tight junction proteins. Conversely, there was no evidence of damage of epithelial cells nor an increase in their proliferation. Of note, our results show that this intestinal inflammatory model is induced independently of the presence of microbiota. On the other hand, this inflammatory process affects intestinal physiology by decreasing protein absorption, increasing neutrophil replacement, and altering microbiota composition with a decrease in the diversity of cultivable bacteria.

Keywords: neutrophil turnover, innate immunity, epithelium permeability, germ free, tight junctions

\section{INTRODUCTION}

Inflammatory bowel diseases (IBD), such as Crohn's disease (CD) and ulcerative colitis (UC), have emerged as a major health problem worldwide (1). A highly prevalent characteristic in IBD patients is the malfunction or loss of the integrity of the intestinal barrier, which can be observed as an increase in permeability and epithelial damage (2) and, in some cases, accompanied by decreased expression or redistribution of tight junction proteins, including occludins, claudins, and junctional adhesion molecules (3). A central factor associated with IBD is the intestinal microbiota since 
many studies have revealed that patients with IBD have a microbiota composition different from that of healthy individuals, characterized by a reduction in butyrate-producing obligate anaerobes from the Firmicutes phylum and a large increase in facultative anaerobes from the Proteobacteria phylum (4). However, it remains unclear if microbial dysbiosis contributes to or is a consequence of intestinal inflammation. One key aspect of IBD is that the intestinal mucosa is damaged by an increase in number and activity of neutrophils (5). Neutrophils not only secrete a variety of tissue-damaging factors, including reactive oxygen species, pro-inflammatory lipid mediators, and proteases, but may also negatively influence tissue repair (6). Furthermore, it has been shown that neutrophils in patients with IBD display delayed apoptosis (7), thus promoting tissue damage. Indeed, in UC, the extent of neutrophil infiltration correlates with the severity of the disease and is included in the UC severity scoring system (8). In contrast, some reports attribute beneficial properties to neutrophils during IBD $(9,10)$. However, it is not clear if these contradictions are due to different experimental strategies or to different neutrophil subsets involved in IBD development (5).

Importantly, many studies of IBD patients are conducted retrospectively, resulting in the inability to determine if the observed gut alterations were a causal event in the etiopathogenesis of IBD or merely the consequence of profound changes in physiology due to the ongoing inflammatory response. In this sense, rodent IBD models have considerably contributed to the understanding of the disease, but the exact contributions of the different risk factors are still unknown as well as the molecular mechanisms responsible for the observed physiological alterations. In this context, complementary approaches to those used until now, difficult to carry out in rodents, such as live imaging and high-throughput screening, can be performed using alternative animal models.

We proposed the use of zebrafish (Danio rerio) as an alternative model to study intestinal inflammation to complement the already available rodent models. It is important to highlight that zebrafish have a conserved intestinal architecture and physiology with mammals in addition to all classic immune cell types (11). Moreover, in some respects, zebrafish show comparative advantages with respect to rodents, such as optical transparency and an increasing number of transgenic lines with labeling of specific cell types. The combination of these two features allow the study of intestinal inflammation process in vivo, in real time, in the whole animal without any surgery and with labeled cell types of interest. Previously, we developed a strategy to induce intestinal inflammation based on the intake of a diet composed mainly of soybean meal and quantification of the inflammation process by neutrophil presence (12). Characterization of the leukocyte response indicates that the inflammatory process triggered in this way is T cell-dependent, and the transcription level of key cytokines suggest a $\mathrm{Th}_{17}$ profile (13). In the present study, we evaluated the effect of the soybean meal-based diet on other critical aspects, such as the integrity of the intestinal epithelium barrier, tight junction protein expression, protein absorption, intestinal neutrophil turnover, and intestinal bacterial microbiota composition. Our findings show that the intestinal inflammation triggered in this manner is characterized by an increase in epithelial permeability and changes in mRNA levels of tight junction proteins; however, epithelial cell damage was observed. Importantly, this intestinal inflammation is induced independently of the presence of microbiota. Finally, as a consequence of the inflammatory process, we observed a decrease in protein absorption capacity, an increase in neutrophil turnover, and a modification of the bacterial microbiota composition with a decrease in the diversity of cultivable bacteria.

\section{RESULTS}

\section{Intestinal Inflammation Induced by Soybean Meal Increases Intestinal Permeability, Alters Tight Junction Protein mRNA Levels, and Decreases Protein Absorption}

In a prior work, we demonstrated how ingestion of a soybean meal-based diet induces a $\mathrm{T}$ cell-dependent intestinal inflammation process in zebrafish larvae $(12,13)$. An important and uncharacterized aspect in this intestinal inflammation model is the integrity of the epithelial barrier. We took advantage of the TgBAC(cdn15la-GFP)pd1034 transgenic line, which expresses a fluorescently labeled Claudin 15 tight junction protein, labeling the contour of enterocytes (14) to detect any alterations. To determine changes in permeability, we performed an in vivo assay, where fluorescently labeled dextran was directly introduced into the gut by microgavage, and 30 min later, the dye distribution was determined. In control larvae, dextran remained in the gut lumen (Figure 1A), whereas in inflamed larvae, dextran breached the intestinal epithelium and diffused into the circulatory system (Figure 1B). Quantification of the normalized mean fluorescence in the trunk, an area where no fluorescence should be observed, indicated that the inflamed gut had increased permeability (Figure 1C). Next, we explored the possibility that the observed increase in permeability was related to changes in the level of proteins that are members of the tight junction complex; thus, we evaluated the mRNA levels of claudin (cldn) 3a, 3d, 7, 8, 11, 15b1, 15b2, 29, 30d, 31 , and 32a; occludin (ocln) $B$ and tight junction proteins (tjp) $1 b 1$ and $1 b 2$ in the gut. Our results indicated that mRNAs encoding Tjp1b1, Tjp1b2, and Cldn15b1 were significantly increased in inflamed larvae compared to controls (Figure 1D). In contrast, transcripts encoding Cldn 3a, 7, 11, 31, 32a, and Ocln b significantly decreased their level in inflamed larvae. We detected no significant changes in mRNA levels of Cldn $3 \mathrm{~d}, 8,15 \mathrm{~b} 2,29 \mathrm{a}$, or $30 \mathrm{~d}$ (Figure 1D). We then analyzed if the ingestion of soybean meal would induce epithelial damage. We prepared transverse histological sections from the midintestine and evaluated the brush border integrity by immunofluorescence. Our results show that both control and inflamed guts had the same amount of brush border interruptions with an average of two per slide (Figures 1E,F,M), interruptions that coincide with the presence of a goblet cell that had released its mucus content into the intestinal lumen (Figures $\mathbf{1 E}-\mathbf{H}, \mathbf{K}, \mathbf{L}$ ). Due to data 
reporting an increase in the amount of mucus during intestinal inflammation (15), we evaluated the number of mucus ${ }^{+}$cells (i.e., goblet cells) in control and inflamed larvae and found that the latter displayed significantly more goblet cells, 32 cells per slide per larva, in contrast to control larvae, which showed 25 per slide per larva (Figures 1G,H,N). Finally, we compared the expression pattern of GFP, which represents Claudin 15, between control and inflamed larvae, and no differences were observed (Figures 1I,J). In summary, these results show that ingestion of soybean meal increases intestinal permeability, alters mRNA levels of several tight junction proteins, and increases the number of goblet cells but does not lead to epithelial histopathology.

To analyze if soybean meal-induced inflammation alters intestinal function, we examined one of the key functions of the intestinal epithelium: protein absorption. We incubated larvae with horse peroxidase (HRP) for $2 \mathrm{~h}$ as the sole protein source and then fixed to histochemically detect HPR within the apical region of specialized enterocytes in the midintestine (16). In the control situation, robust HRP staining was detected in a section of the gut, covering an average of 4 somites (Figures 10,Q). Likewise, in the inflamed gut, HRP stain was also present but in a narrow region, comprising an average of 2 somites (Figures 1P,Q). We also evaluated if the inflammation process affected the proliferation rate of intestinal epithelial cells and, with this, epithelial replacement. To this end, we incubated larvae with the thymidine nucleoside EdU for $16 \mathrm{~h}$ and then detected its incorporation into newly synthesized nuclear DNA. The results showed that the number of proliferating cells detected in the guts of control larvae was indistinguishable from that observed in the guts of inflamed larvae (Figures 1R-T). Thus, our results suggest that the inflamed guts decrease their protein absorption capacity but retain normal proliferation rates, indicating that the epithelium is not undergoing inflammationinduced hypertrophy.

\section{Intestinal Inflammation Induced by Soybean Meal Occurs Under Germ-Free Conditions}

At present, there are intestinal inflammatory models where the microbiota is a key player in the induction of inflammation as is the case of the DSS model $(17,18)$. Thus, to determine the function of the intestinal microbiota in the induction of the inflammatory process, we performed the feeding protocol under germ-free conditions (16). The results show that the number of neutrophils present in the intestine increased in conventionally bred larvae, corroborating what was previously described (12) in both control and inflamed larvae with an average of 4 neutrophils in the control condition and 10 in the inflamed condition (Figures 1U,V,Y). Similarly, in a germ-free environment, the number of neutrophils present in the intestines of control larvae reached an average of 6 neutrophils and in inflamed larvae 11 neutrophils (Figures $\mathbf{1 W}-\mathbf{Y}$ ), indicating that an inflammatory process was underway. Thus, these results reveal that our inflammatory model is independent on the presence of intestinal microbiota because, in germ-free larvae, inflammation is still triggered.

\section{Intestinal Inflammation Induced by Soybean Meal Increases Neutrophil Turnover}

In a previous report, we demonstrated that the soybean mealbased diet induced a persistent neutrophil infiltration into the midintestine of zebrafish larvae (12). To understand the dynamics of neutrophil recruitment to the gut, we took advantage of the $\operatorname{Tg}(\operatorname{mpxI}: \text { Dendra2 })^{\mathrm{uwm} 4 \mathrm{Tg}}$ transgenic line, in which the fluorescence of transgenic neutrophils can be switched from green to red after photoconversion of the Dendra2 protein (19). Thus, we induced the inflammatory process and performed two photoconversions, every $24 \mathrm{~h}$, quantifying neutrophils before and after each photoconversion (Figure 2A). Green neutrophils in the gut represent those newly arrived during a period of $24 \mathrm{~h}$, and red neutrophils outside the gut are those that left the intestine during a period of $24 \mathrm{~h}$ (Supplementary Figures 1A-L). At $0 \mathrm{~h}$ post-photoconversion (hpc), we observed an average of 10 neutrophils in control larvae and 19 in inflamed larvae (Figure 2B). At $24 \mathrm{hpc}$, we found that, in control larvae, the number of total neutrophils remained similar with 11 present in the gut (Figure 2B). Importantly, an average of 3 neutrophils were replaced (Figure 2C), representing 30\% of total neutrophils. Likewise, in the case of inflamed larvae, 21 neutrophils were present in the gut, and 6 neutrophils were replaced (Figures 2B,C), corresponding to $29 \%$ of total neutrophils in the gut. A similar situation in control and inflamed larvae was observed at $48 \mathrm{hpc}$ where 4 and 7 neutrophils were replaced, respectively (Figure 2C). Although the percentage of replacement was similar in both conditions, the total amount of substituted neutrophils in the inflamed condition was at least double compared to those changed in the control condition at 24 and $48 \mathrm{hpc}$ (Figure 2C). These results show that neutrophil presence in the intestine is dynamic and that neutrophils are replaced continually and in greater numbers in the inflamed condition. On the other hand, it appears that, during inflammation, the observed neutrophil increase was due to a rise in cells recruited to the intestine rather than caused by the proliferation of neutrophils that were already present there. To test this hypothesis, we photoconverted the two regions were neutrophils are mainly located in zebrafish larvae: the head (where more differentiated neutrophils are present) and the caudal hematopoietic tissue (CHT, were non-activated or more undifferentiated neutrophils are located). Then, after $24 \mathrm{hpc}$, we quantified red neutrophils present in the gut (Figure 2D). We found that, in the inflammatory condition, an average of 4 head-red neutrophils and 6 CHT-red neutrophils were in the gut (Figures 2E-G). In the case of the control condition, as expected, the replaced neutrophils were recruited from the head and CHT (Supplementary Figures 1M-P). This result confirms that, during inflammation, the new neutrophils in the intestine are recruited from other tissues.

Next, we hypothesized that neutrophils that leave the intestine during inflammation have a different destination compared 

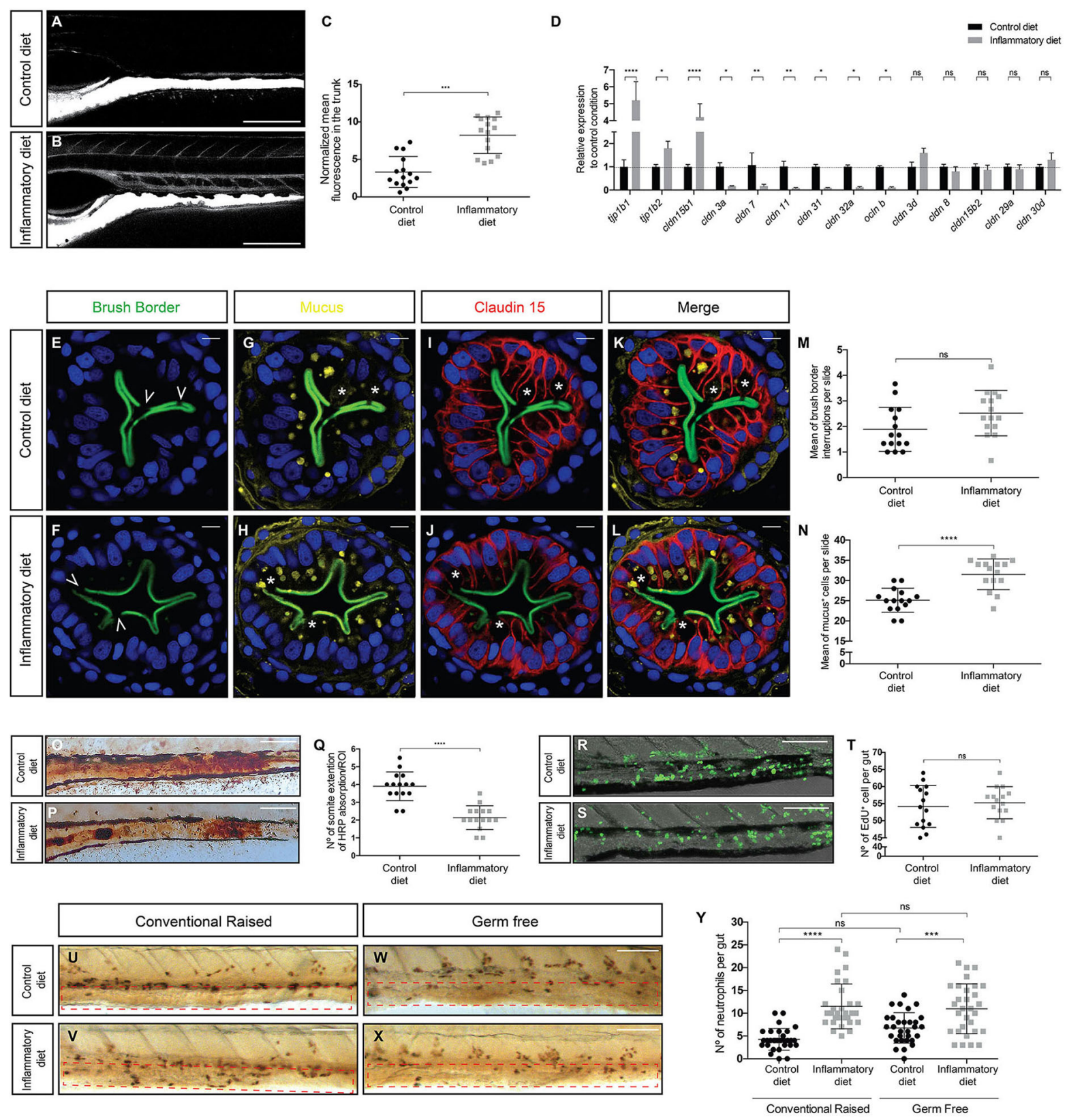

FIGURE 1 | Intestinal inflammation induced by soybean meal alters intestinal physiology and is independent of the presence of microbiota. (A,B) Lateral view of the mid-intestine of 9 dpf larvae showing the diffusion of dextran in control (A) and inflamed (B) larvae. Scale bar, 200 um. (C) Normalized dextran fluorescence quantification in the trunk of control and inflamed larvae. (D) Relative mRNA expression of several tight junction proteins. All data was normalized against $r p / 13 a$ and compared to the control condition (dotted line). (E-L) Transversal cryosection of the midintestine of $9 \mathrm{dpf}$ control and inflamed larvae. (E-J) Immunofluorescence labeling the brush border $\mathbf{( E , F ) , ~ m u c u s ~} \mathbf{( G , H )}$, and Claudin $15 \mathbf{( I , J ) ; ~ n u c l e i ~ w e r e ~ s t a i n e d ~ w i t h ~ D A P I ~ ( b l u e ) . ~ ( K , L ) ~ M e r g e ~ o f ~ t h e ~ f o u r ~ c h a n n e l s ~ i n ~ c o n t r o l ~ a n d ~ i n f l a m e d ~}$ larvae. Scale bar, 5 um. (M,N) Quantification of brush border interruptions (white arrowheads in $\mathbf{E , F}$ ) and goblet cells (white asterisks in $\mathbf{G}$ and $H)$. (O,P) Representative images of the area of the midintestine showing HRP absorption in control and inflamed larvae. Scale bar, $100 \mathrm{um}$. (Q) Quantification of the area covered by HRP in the midintestine of control and inflamed larvae. (R,S) Representative images showing the number of cell that proliferate during $16 \mathrm{~h}$ in control and inflamed larvae. Scale bar, 100 um. (T) Quantification of EdU' cells in the midintestine per larva. (U-X) Lateral view of the midintestine showing immunohistochemistry against the neutrophil marker Mpx on those conventionally raised. The area quantified is delimited by the red dotted rectangle (U,V) and germ-free larvae (W,X). Scale bar, 100 um. (Y) Quantification of the amount of neutrophils present in the midintestine in conventionally raised and germ-free larvae. Permeability, immunofluorescence, protein absorption, proliferation assay, and immunohistochemistry were performed at least in three biological replicates with 15 larvae of 9 dpf per condition. Statistical analysis was performed using the Mann-Whitney U-test, ${ }^{* \star *} p<0.001,{ }^{* \star \star \star} p>0.0001$. RT-qPCR was performed at least in three biological replicates with 100 intestines from $9 \mathrm{dpf}$ larvae per condition. Statistical analysis was analyzed using one-way ANOVA and Tukey multiple comparison test. ns, non-significant, ${ }^{*} p<0.05$, ${ }^{\star \star} p<0.01$, and ${ }^{\star \star \star *} p<0.0001$ 


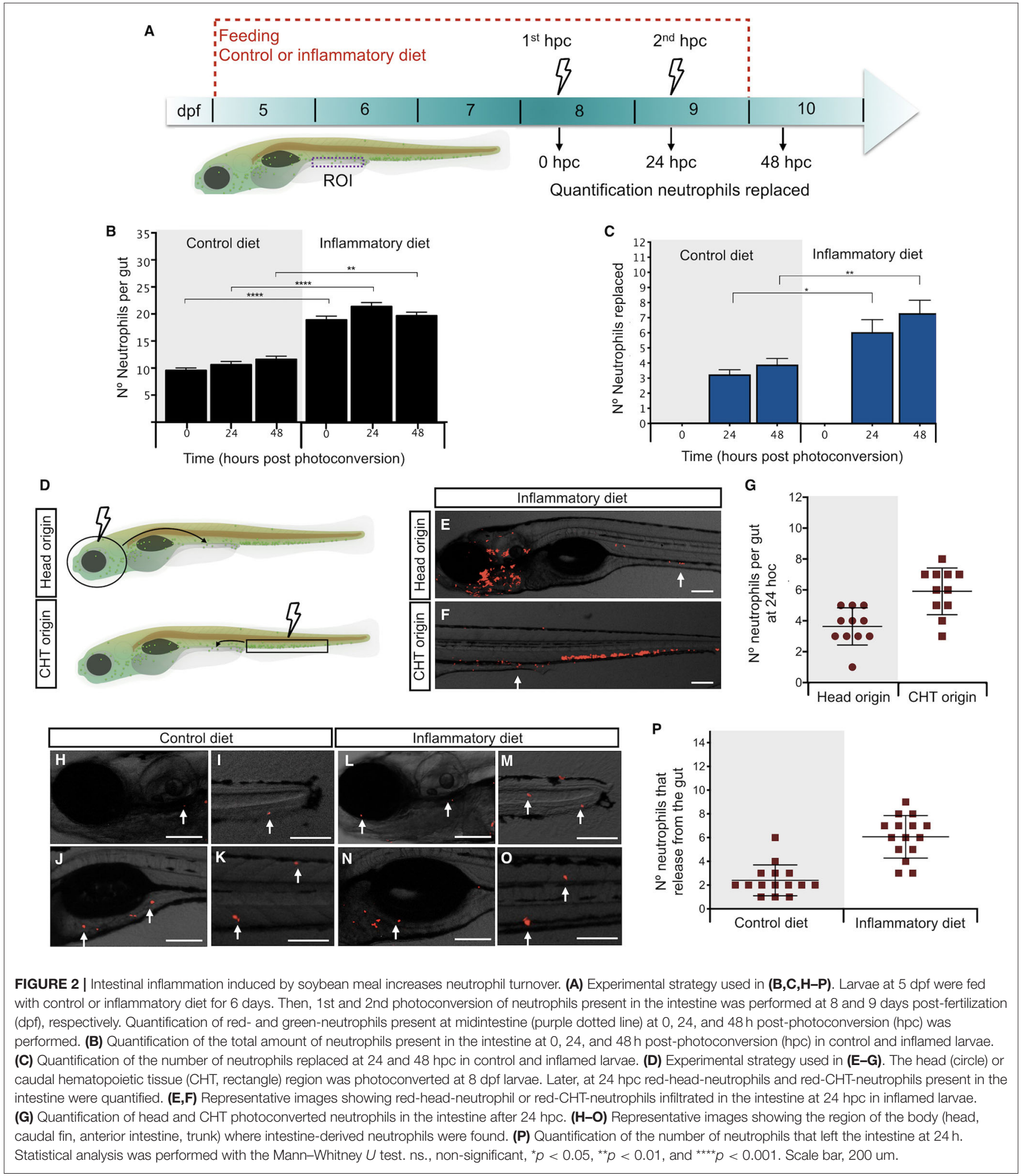

to controls; thus, we decided to determine their location at 24 hpc. After applying the strategy described in Figure 2A, we observed that, in the control and inflammatory conditions, neutrophils that were previously present in the gut were now located at the head, tail, somites, caudal hematopoietic tissue (CHT), and anterior intestine (Figures 2H-P), suggesting that there is no preferred region for each specific group. 


\section{Intestinal Inflammation Induced by Soybean Meal Results in Modification of Dominant and Cultivable Bacterial Microbiota}

To explore the microbiomes of inflamed and control larvae, independent of culturing, we performed 16S rRNA amplicon sequencing on individual larval intestines. When we compared the resulting bacterial profiles, we found that overall control and inflamed fish did not have significantly different distributions of the Shannon index, a measure of phylogenetic diversity ( $\alpha$-diversity), or non-metric multidimension scaling (NMDS) using the Jaccard similarity coefficient ( $\beta$-diversity). Of the 186 genera detected in larvae gut, 18 changed significantly in relative abundance, depending on the diet, fishmeal or soybean meal (Figure 3A). All three of our analytic techniques showed that inflamed larvae had a significantly increased relative abundance of the Bacilli family compared to control larvae. Specifically, in the Bacilli family, QIIME analysis showed that Staphylococcus was significantly increased (Figure 3B). Interestingly, inflamed larvae also had increased relative abundance of an unclassified genus in the Bifidobacteriaceae family. All other significant differences were found in the Proteobacteria phylum, showing that inflamed larvae had increased relative abundance of Brevundimonas and Alcanivorax and a decreased relative abundance of Plesiomonas, Shewanella, and an unclassified genus in the Aeromonadaceae family (Figures 3A,C-E). Next, we determined if the soybean meal alters the composition of intestinal cultivable bacterial microbiota. For this purpose, we cultured control and inflamed guts and sequenced the $16 \mathrm{~S}$ rRNA genes of cultured bacteria. Similar bacterial counts were detected in control and inflamed intestine, $10^{4.4}$ and $10^{4.5}$ CFU/larvae, respectively ( $p<0.05$, Mann-Whitney test). We recovered Proteobacteria, Actinobacteria, and Firmicutes with similar relative abundance in inflamed and control larvae $(p<0.05)$, with a marked dominance of the Proteobacteria phylum in both groups (Figures 3F,I). More differences were observed at the genus and species level although no significant differences were observed in relative abundances between both groups (Figures $\mathbf{3 G}, \mathbf{H}, \mathbf{J}, \mathbf{K}$ ). Only one abundant Aeromonas was shared between groups. In the control group, we identified different species of Plesiomonas and Pseudomonas (Supplementary Table 1). Acidovorax sp., uncultured Kocuria, and Aeromonas hydrophila were also detected. As we observed in the direct microbiota analysis, Aeromonas and Plesiomonas were most prevalent in control larvae. In inflamed larvae, we identified Kocuria sp., uncultured Roseateles, Bacillus sp., Vibrio cholerae, Paenibacillus, Arthrobacter sp., and different Aeromonas and Shewanella species. These results showed that inflamed larvae compared to control larvae harbored different bacterial species (Supplementary Table 1).

\section{DISCUSSION}

In the present work, we characterize the effects induced by the ingestion of a soybean meal diet on intestinal epithelial barrier integrity and microbiota composition as well as the role of gut microbiota in inducing the inflammation process. We found that soybean meal increases intestinal permeability, presumably by altering the composition of the tight junction protein complex but without leading to overt pathology of the brush border or to epithelial hyperplasia. Moreover, we found that the extent of inflammation generated in this manner is sufficient to impair intestinal protein absorption capacity and increase neutrophil turnover in the intestine. Importantly, the inflammation shown is independent of the presence of commensal bacteria even though we show soybean meal-induced intestinal bacterial population changes. These results suggest that, in our intestinal inflammation model, inflammation is a primary response to the presence of soybean meal and a driver of bacterial microbiota composition modification although we cannot exclude a possible effect of the diet in the microbiota composition.

Increased intestinal permeability is a main feature of many diseases, including IBD (20) as well as several mouse model of enteritis (21-23). In our case, we found increased permeability together with changes in transcript abundance of several tight junction proteins. Numerous reports have demonstrated the effect of tight junction alterations in intestinal permeability. In a mouse model of $\mathrm{T}$ cell-induced acute diarrhea, intestinal permeability was associated with the rearrangement of cellular distribution of occludin and, to some extent, proteins of the junctional adhesion molecules family (24). Likewise, it is reported that patients with active Crohn's disease or ulcerative colitis and impaired intestinal permeability have decreased expression of occludin as well as claudin $4,5,7$, and 8 , whereas claudin 2 is strongly upregulated (25). In agreement with this evidence, we observed that occludin $\mathrm{b}$ and claudin 7 were downregulated as were other claudins, such as claudin $3 \mathrm{a}, 11,31$, and $32 \mathrm{a}$. In addition, a previous report in another fish species, turbot, indicated that the ingestion of a soybean meal-based diet increased intestinal permeability (26). By analyzing histological sections of the intestine, the authors found that intercellular junctions were disrupted in soybean meal-fed fish soybean meal, resulting in an increased intercellular space. Likewise, in another previous study where weaned piglets were fed a soybean-extracted lectin (agglutinin), Zhao et al. found that the expression of intestinal epithelial ZO-1 declined, leading to increased intestinal permeability (27). Thus, considering these reports and the fact that soy protein does not induce intestinal inflammation or epithelial barrier dysfunction in zebrafish, but saponin, a glucoside present in soybean, does (12), we hypothesize that the mechanism by which a soybean meal diet induces intestinal inflammation is through compounds, such as lectins and saponins, that impair the expression of several proteins that form part of the tight junction complex, thus increasing intestinal permeability.

One important aspect of our intestinal inflammation model is that it is induced independently of the presence of microbiota. In general, in mouse models of colitis where the epithelial barrier is severely disrupted due to chemical compounds or genetic defects, such as in the DSS-colitis model $(28,29), \mathrm{N}$ cadherin dominant negative mice (30), Muc1/2-/- mice $(31,32)$, $\mathrm{Mdrla}^{-/-}$mice (33), or in those animals where the immune response is compromised due to the genetic background, such 


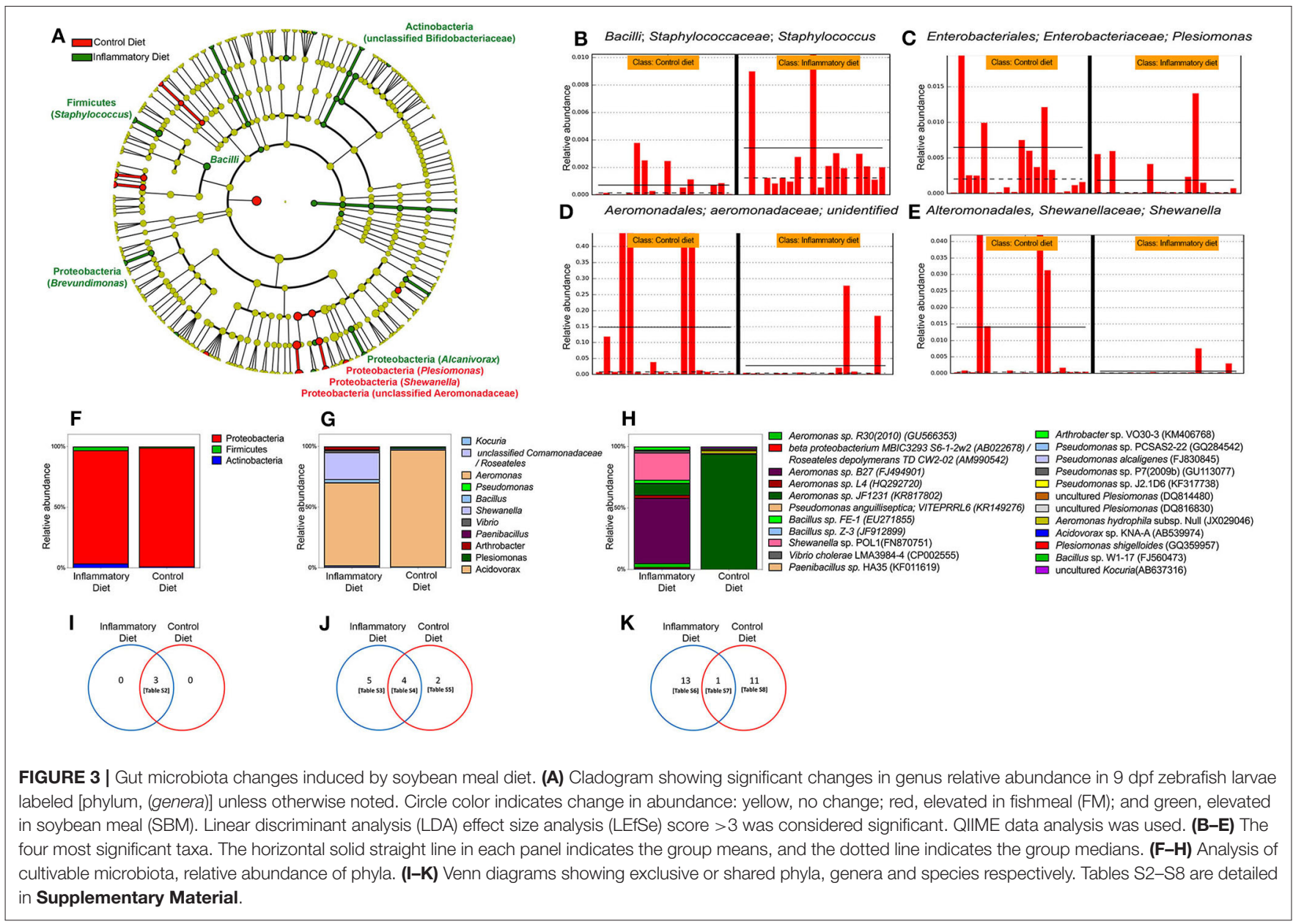

as in $\mathrm{IL} 10^{-/-}$and $\operatorname{Stat} 3^{-/-}$mice $(34,35)$, inflammation is absent when animals are maintained under germ-free conditions. Of note, when animals are removed from their gnotobiotic condition, mice rapidly develop an inflammatory process (36). In these cases, the commensal microbiota induce the inflammation process, mainly due to the gross abnormalities of the gut epithelial barrier that allow bacteria to be present in the lamina propria or due to a defective mucosal T cell response (21). This requirement for microbiota to drive the inflammatory process is also observed in a zebrafish genetic model of spontaneous intestinal inflammation (22). In contrast, we observed an increased neutrophil number in the intestine of animals with normal genetic background when maintained under germ-free conditions, indicating that soybean meal is directly responsible for triggering the inflammatory process. The observed differences in microbiota between the soybean meal-fed and control larvae were subtle and likely arose as a consequence of the differences in the intestinal environment between these two groups with no significant differences in phylogenetic diversity. Using three independent analytic approaches, we showed that bacteria in the Bacilli class were increased in soybean meal-fed larvae, in particular Staphylococcus. Inflammation causes physiological changes in the intestine, such as availability of oxygen and other electron acceptors, that can drive overgrowth of certain pathogenic bacterial groups, including Proteobacteria, and create positive feedback loops of dysbiosis (37). Interestingly, the Proteobacteria genera Shewanella was decreased in soybean meal-fed larvae. Previous work in zebrafish has shown that Shewanella may play a protective role against inflammation (38). In our analysis, significant shifts in particular bacterial genera, rather than the overall community composition, were observed, and whether these shifts are reproducible in response to the mentioned intestinal physiological parameters or others remains to be determined.

On the other hand and in agreement with this study, previous works have shown that the inclusion of soybean meal in the fish diet did not alter intestinal bacterial counts (39). Of note, the shift in observed cultivable species has been correlated with the inclusion of soybean meal and not with the intestinal inflammation condition (39). In addition, a dominant sequence identified as Bifidobacterium has been detected in the pelleted feed of Atlantic salmon containing 10\% soybean meal (40). In our study, similar sequences (uncultured Bifidobacteriaceae) were more abundant in soybean meal-fed larvae, suggesting that this vegetable component could select this microorganism in feed and/or in our soybean meal-fed larvae. Further analyses must be 
performed in order to elucidate whether these bacterial shifts are related to soybean meal inclusion or intestinal inflammation.

The soybean meal diet induced physiological changes in the intestine that could be observed as a decrease in protein absorption and increase in neutrophil turnover and dysbiosis. Malabsorption of macronutrients, such as fat, and micronutrients, such as Vitamin B12, Vitamin D (17), and trace elements (18), has been reported in patients with Crohn's disease. The most likely reason for this is the loss of intestinal absorptive surface and malfunction of the mucosa. In the case of neutrophil infiltration in the intestine, we observed that not only the number of intestinal neutrophils is increased, but also their daily replacement. In agreement with this result is the fact that the extent of neutrophil infiltration correlates with the severity of ulcerative colitis (8), and patients with active ulcerative colitis display an increased neutrophil-to-lymphocyte ratio in the blood (41). Neutrophil contribution to the pathogenesis of IBD remains controversial; some studies describe a beneficial role, and others report detrimental effects $(10,42,43)$. One explanation for this fact could be that different neutrophils subsets are involved in active IBD (9). Notably, massive neutrophil transmigration across the intestinal epithelial barrier has been shown to alter levels of tight junction proteins, thereby increasing epithelial permeability and facilitating the arrival of more neutrophils to the gut, thus triggering an uncontrolled positive feedback amplification loop leading to tissue damage and resolution delay (45).

Further investigation is needed to clearly understand the specific weight of each risk factor in the etiopathogenesis of IBD and the physiological effects that they induced in the gut. Thus, based on the conserved intestinal and immune response between mammals and zebrafish, our intestinal inflammatory model is a suitable tool to contribute to deciphering the mechanisms underlying this complex disease and to provide a rational basis for the development of more effective and targeted therapeutic interventions.

\section{MATERIALS AND METHODS}

\section{Ethics Statement}

All animal handling procedures were approved by the "Committee of Animal Bioethics of the Universidad Andres Bello," Certificate number 007/2016.

\section{Zebrafish Husbandry}

Adult zebrafish were maintained at the fish facility of the Universidad Andres Bello, following standard protocols (46). TgBAC(cdn15la-GFP)pd1034 (14) and Tg(mpxI:Dendra2) ${ }^{\text {uwm } 4 \mathrm{Tg}}$ (19) embryos were obtained by natural spawning and maintained at $28.5^{\circ} \mathrm{C}$ in E3 medium $(5 \mathrm{mM}$ $\left.\mathrm{NaCl}, 0.17 \mathrm{mM} \mathrm{KCl}, 0.33 \mathrm{mM} \mathrm{CaCl}_{2}, 0.33 \mathrm{mM} \mathrm{MgSO}_{4}, \mathrm{pH} 7.0\right)$.

\section{Feeding Strategy}

Experimental feeding was conducted as was previously described by Hedrera et al. (12) with some modification. Briefly, 30 larvae were maintained in $100 \mathrm{ml}$ of aquarium water and fed with a fishmeal-based diet (from now on control diet) or a soybean meal-based diet (from now on inflammatory diet) (Supplementary Table 9) twice a day from 5 to 8 days postfertilization (dpf). After the feeding strategy, larvae were changed to fresh aquarium water and left for $19 \mathrm{~h}$ without food to promote intestinal emptying.

\section{Photoconversion and in vivo Neutrophil Quantification}

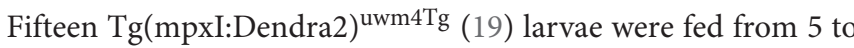
9 days post-fertilization ( $\mathrm{dpf}$ ) according to the protocol described above. At 8 and $9 \mathrm{dpf}$, larvae were anesthetized with $0.017 \%$ tricaine and embedded in $2 \%$ methylene cellulose to performed neutrophil photoconversion. The midintestine was incised with ultraviolet light $(350-400 \mathrm{~nm})$ at $90 \%$ potency for $1 \mathrm{~min}$ of exposure under a fluorescence microscope (Olympus BX51) using minimum aperture diaphragm. Then the number of green and red neutrophils present in the midintestine were quantified right after photoconversion and at 8, 9, and $10 \mathrm{dpf}$. Neutrophil replacement was determined according to the number of red neutrophils found outside the intestine and green neutrophils present the intestine (Supplementary Figure 1).

\section{Histological Sections and Immunolabeling}

Nine dpf larvae were anesthetized and fixed in PFA (4\% paraformaldehyde in phosphate buffered saline) overnight. Larvae were embedded in $1.5 \%$ low melting agarose, dehydrated in $30 \%$ sucrose solution overnight at $4^{\circ} \mathrm{C}$ and cut in into $30 \mu \mathrm{m}$ slices using a Leica CM1510S cryostat. Cryosectioned tissue was refixed with PFA for $20 \mathrm{~min}$, followed by successive washes in $1 \%$ DMSO. Tissue was incubated in blocking solution (1\% BSA, 1\% DMSO, 5\% Goat Serum) for $1 \mathrm{~h}$ and incubated overnight with either anti-brush border (Abcam) or anti GFP (ThermoFisher Scientific). Secondary antibody Alexa 488, Alexa 594, or Alexa 647 (Molecular Probes) was used. Mucus staining was performed with WGA (Molecular Probes). Sections were mounted in SlowFade (Life Technologies) and analyzed under an Olympus FluoView FV1000 Spectral Confocal Microscope. The mean of brush border interruptions and mucus positive cells per slide in each larva was plotted. Fifteen larvae were analyzed per condition in three independent experiments.

\section{Permeability Assay}

Oral microgavage was performed as previously described (47) with minor modifications. Briefly, 15 larvae exposed to the experimental feeding strategy were anesthetized, mounted in $2.5 \%$ methylcellulose (Sigma) and gavaged with $\sim 5 \mathrm{~nL}$ of $1 \%$ solution of FITC-dextran (4,000 MW; Sigma). After $15 \mathrm{~min}$, larvae were imaged under an Olympus FluoView FV1000 Spectral Confocal Microscope. Images were processed using ImageJ software to quantify the fluorescence density in the intestine and in the somites dorsal to this region. As a control, a background region was measured outside of the fish and subtracted from the density quantified in the somite region. All values were normalized to the average value from each control group. 


\section{Quantitative Polymerase Chain Reaction (qPCR)}

One hundred intestines per diet were processed for total RNA extraction using NucleoSpin ${ }^{\circledR}$ RNA Xs (Macherey-Nagel) according to the manufacturer's instructions, and RNA integrity was corroborated by denaturing agarose electrophoresis. cDNA was synthesized from $2.5 \mu \mathrm{g}$ of total RNA using SuperScript II Reverse Transcriptase and Oligo-dT primers (ThermoFisher Scientific). Primer sequences are indicated in Supplementary Table 10. qPCR was performed as was previously described (12). The mean Ct values from each sample were normalized against the mean $\mathrm{Ct}$ value of a reference gene $(r p l 13 a)$. All experiments were done in triplicate and with three biological replicates.

\section{Proliferation Assay}

Larvae were exposed to the nucleotide analog 5-ethynyl-2' deoxyuridine (EdU) as previously described (48) with some modifications. Larvae were immersed in $100 \mu \mathrm{g} / \mathrm{ml} \mathrm{EdU}$ (Invitrogen) with $0.5 \%$ DMSO for $16 \mathrm{~h}$ and fixed overnight at $4{ }^{\circ} \mathrm{C}$ in $4 \%$ PFA. Larvae were then processed using the ClickiT EdU Imaging Kit (Invitrogen). EdU-labeled nuclei within the intestine were quantified in at least 30 larvae per condition in three independent experiments.

\section{Protein Absorption Assay}

Protein absorption was measured as previously described (49). Briefly, after $19 \mathrm{~h}$ of starvation following the experimental feeding strategy, larvae were incubated overnight in $2 \mathrm{mg} / \mathrm{ml} \mathrm{HRP}$ (Sigma) and fixed in PFA. HRP activity was detected using Vector HRP substrate kit (Vector Labs) and the area covered by the mark was measured as number of somites.

\section{Gnotobiotic Generation and Feeding Strategy Under Germ-Free Conditions}

Germ-free larvae were generated as previously described $(16,50)$. At $5 \mathrm{dpf}$, germ-free larvae began to be fed with UV-sterilized diets, following the same strategy of conventional feeding. Five larvae were sampled each day, homogenized and incubated in Tryptic Soy Broth without antibiotics for $16 \mathrm{~h}$ to check bacterial growth.

\section{Neutrophil Immunodetection}

Immunohistochemistry was performed as previously described (13), using a myeloperoxidase (Mpx) primary antibody (Genetex) followed by its detection with anti-rabbit peroxidase (Sigma). The number of neutrophils infiltrated in the midintestine was quantified as was previously described $(12,13)$. A minimum of 30 larvae were analyzed per condition in three independent experiments.

\section{Identification of Cultivable Aerobic Gut Microbiota}

Ten intestines per treatment in duplicate were extracted and resuspended in $250 \mu \mathrm{l}$ PBS. 10-fold serial dilutions were plated onto trypticase soy agar and incubated for 3 days at $28^{\circ} \mathrm{C}$ in duplicate. All colonies were isolated, purified, and identified by sequencing the $16 \mathrm{~S}$ rRNA gene, as described below. DNA from each morphologically different colony was extracted. Amplification of the gene coding 16S rRNA gene performed with the primers 27F $5^{\prime}$ - AGAGTTTGAT CCTGGCTCAG-3' and 1492R 5'-ATTTGCTAAAGCGGG AATCT-3' as previously described (51). PCR reactions were performed in a reaction mixture containing $1 \mathrm{X}$ GoTaq ${ }^{\circledR}$ Green Master Mix (Promega, Madison, WI, USA) and 0.25 $\mathrm{pmol} / \mu \mathrm{l}$ of each primer. PCR products were purified and sequenced by Macrogen USA sequencing service. Sequences were edited and cleaned using BIOEDIT software (http:// www.mbio.ncsu.edu/BioEdit/bioedit.html). Each sequence was analyzed to find GenBank sequences with the closest BLAST-N hits.

\section{Microbial DNA Extraction and Sequencing}

Intestines of $9 \mathrm{dpf}$ larvae fed either fishmeal or soybean meal were dissected and flash-frozen in liquid nitrogen. Samples were shipped to University of Oregon to be analyzed. DNA was extracted using the PowerMag Microbiome RNA/DNA isolation kit (MoBio, Cat 27500-4-EP), using the Geno/Grinder 2010 for homogenization and epMotion 5075 TMX (eppendorf) liquid handler, according to the manufacturer's protocol. Amplification was performed on the V4 region of the 16S rRNA genes via PCR using the following primers: F515 (5'-GTGCCAGCMGCCGCG GTAA-3') and R806 (5'-GGACTACHVGGGTWTCTAAT-3') (52). High-throughput sequencing was performed on 37 DNA samples with Illumina HighSeq paired-end 150-bp runs at the University of Oregon Genomics, Cytometry and Imaging Core.

\section{Microbial Bioinformatic Analysis}

Quantitative Insights Into Microbial Ecology (QIIME, version 1.9.1) (53) software was used for demultiplexing and quality filtering of raw sequences. Operational taxonomic unit (OTU) picking was performed using an open reference workflow script with Greengenes 97 (version 13_8) for taxonomy assignment at 97\% similarity. Chimera sequences were identified via Chimera Slyer (54) and removed. With the use of additional filtering, low-read OTUs were also removed (55). Of the 84,505,228 reads analyzed, 84,461,129 reads were used after filtering, resulting in 343 observed OTUs. Diversity, weighted UniFrac principal coordinate analysis (PCoA), and taxa summaries were created through QIIME (core_diversity_analyses.py) with sequencing depth and maximum rarefaction depth set at 300,000. Relative abundance outputs were between $3.52 \times 10^{-7}$ and 97.27\%. Alpha and beta diversity were analyzed using QIIME (compare_alpha_diversity.py \& make_distance_boxplots.py). Multivariable statistical analysis was performed in the LEfSe package (56) with default parameters. To validate QIIME analysis approach and results, raw sequences were analyzed using DADA2 (57) and phyloseq as we described (44).

\section{Statistical Analysis}

Dextran diffusion, immunofluorescence, protein absorption, proliferation, and neutrophil quantification were analyzed using 
Mann-Whitney $U$-test. mRNA expression was analyzed using one-way ANOVA and Tukey multiple comparison test. All analyses were performed using Prim 6 (GraphPad Software). The significance level was set to $P<0.05$ or below.

\section{DATA AVAILABILITY STATEMENT}

All datasets generated for this study are included in the article/Supplementary Material.

\section{ETHICS STATEMENT}

The animal study was reviewed and approved by Ethics Committee from the Universidad Andres Bello, Certificate number $007 / 2016$.

\section{AUTHOR CONTRIBUTIONS}

$\mathrm{CS}, \mathrm{CF}, \mathrm{MC}, \mathrm{PN}, \mathrm{MH}$, and $\mathrm{KG}$ contributed to the conception and design of the study. CS, JG-L, MC, and MH developed the experiments and performed statistical analysis. CF wrote the first draft of the manuscript. All authors contributed to revising the manuscript, reading, and approving the submitted version.

\section{REFERENCES}

1. Colombel JF, Mahadevan U. Inflammatory bowel disease 2017: innovations and changing paradigms. Gastroenterology. (2017) 152:309-12. doi: 10.1053/j.gastro.2016.12.004

2. Soderholm JD, Peterson KH, Olaison G, Franzen LE, Westrom B, Magnusson KE, et al. Epithelial permeability to proteins in the noninflamed ileum of Crohn's disease? Gastroenterology. (1999) 117:65-72. doi: 10.1016/S0016-5085(99)70551-2

3. Lee SH. Intestinal permeability regulation by tight junction: implication on inflammatory bowel diseases. Intest Res. (2015) 13:11. doi: 10.5217/ir.2015.13.1.11

4. Degruttola AK, Low D, Mizoguchi A, Mizoguchi E. Current understanding of dysbiosis in disease in human and animal models. Inflamm Bowel Dis. (2016) 22:1137-50. doi: 10.1097/MIB.0000000000000750

5. Wéra O, Lancellotti P, Oury C. The dual role of neutrophils in inflammatory bowel diseases. J Clin Med. (2016) 5:118. doi: 10.3390/jcm5120118

6. Koh TJ, DiPietro LA. Inflammation and wound healing: the role of the macrophage. Expert Rev Mol Med. (2011) 13:112. doi: $10.1017 /$ S1462399411001943

7. Brannigan AE, O’Connell PR, Hurley H, O’Neill A, Brady HR, Fitzpatrick JM, et al. Neutrophil apoptosis is delayed in patients with inflammatory bowel disease. Shock. (2000) 13:361-6. doi: 10.1097/00024382-200005000-00003

8. Bressenot A, Salleron J, Bastien C, Danese S, Boulagnon-Rombi C, PeyrinBiroulet L. Comparing histological activity indexes in UC. Gut. (2015) 64:1412-18. doi: 10.1136/gutjnl-2014-307477

9. Zhou G, Yu L, Fang L, Yang W, Yu T, Miao Y, et al. CD177+ neutrophils as functionally activated neutrophils negatively regulate IBD. Gut. (2018) 67:1052-63. doi: 10.1136/gutjnl-2016-313535

10. Natsui M, Kawasaki K, Takizawa H, Hayashi SI, Matsuda Y, Sugimura K, et al. Selective depletion of neutrophils by a monoclonal antibody, RP-3, suppresses dextran sulphate sodium-induced colitis in rats. J Gastroenterol Hepatol. (1997) 12:801-8. doi: 10.1111/j.1440-1746.1997.tb00375.x

11. Brugman $\mathrm{S}$. The zebrafish as a model to study intestinal inflammation. Dev Comp Immunol. (2016) 64:82-92. doi: 10.1016/j.dci.2016.02.020

\section{FUNDING}

This study was supported by Millennium Science Initiative, Ministry of Economy, Tourism of Chile under Grant Nucleus in the Biology of Intestinal Microbiota (CF and PN), FONDECYT 1171199 (CF), FONDECYT 1181499 (PN), NIH award 1P01GM125576 (KG), and CONICYT Scholarship 21140604 (CS).

\section{ACKNOWLEDGMENTS}

We thank Dr. Michel Bagnat who kindly provided us the transgenic line TgBAC(cdn15l-GFP)pd1034. Dr. Juan Carlos Rivera, who helped in the process of standardization of histological sections and Fernando Lazcano for technical assistance. We also thank the students from the University of Oregon Bioinformatics and Genomics Masters Program for microbiota sequencing analysis assistance.

\section{SUPPLEMENTARY MATERIAL}

The Supplementary Material for this article can be found online at: https://www.frontiersin.org/articles/10.3389/fimmu. 2020.01330/full\#supplementary-material

12. Hedrera MI, Galdames JA, Jimenez-Reyes MF, Reyes AE, Avendaño-Herrera $\mathrm{R}$, Romero J, et al. Soybean meal induces intestinal inflammation in zebrafish larvae. PLoS ONE. (2013) 8:e69983. doi: 10.1371/journal.pone.0069983

13. Coronado M, Solis CJ, Hernandez PP, Feijóo CG. Soybean meal-induced intestinal inflammation in zebrafish is $\mathrm{T}$ celldependent and has a Th17 cytokine profile. Front Immunol. (2019) 10:610. doi: 10.3389/fimmu.2019.00610

14. Alvers AL, Ryan S, Scherz PJ, Huisken J, Bagnat M. Single continuous lumen formation in the zebrafish gut is mediated by smoothened-dependent tissue remodeling. Development. (2014) 141:1110-19. doi: 10.1242/dev.100313

15. Pullan RD, Thomas GA, Rhodes M, Newcombe RG, Williams GT, Allen A RJ. Thickness of adherent mucus gel on colonic mucosa in humans and its relevance to colitis. Gut. (1994) 35:353-9. doi: 10.1136/gut.35.3.353

16. Caruffo M, Navarrete NC, Salgado OA, Faúndez NB, Gajardo MC, Feijóo CG, et al. Protective yeasts control $V$. anguillarum pathogenicity and modulate the innate immune response of challenged zebrafish (Danio rerio) larvae. Front Cell Infect Microbiol. (2016) 6:217. doi: 10.3389/fcimb.2016.00127

17. Margulies SL, Kurian D, Elliott MS, Han Z. Vitamin D deficiency in patients with intestinal malabsorption syndromes - think in and outside the gut. J Dig Dis. (2015) 16:617-33. doi: 10.1111/1751-2980.12283

18. Geerling BJ, Stockbrügger RW, Brummer RJM. Nutrition and inflammatory bowel disease: an update. Scand J Gastroenterol Suppl. (1999) 33:95105. doi: 10.1080/003655299750025615

19. Yoo SK, Huttenlocher A. Spatiotemporal photolabeling of neutrophil trafficking during inflammation in live zebrafish. J Leukoc Biol. (2011) 89:6617. doi: $10.1189 /$ jlb.1010567

20. Ahmad R, Sorrell MF, Batra SK, Dhawan P, Singh AB. Gut permeability and mucosal inflammation: bad, good or context dependent. Mucosal Immunol. (2017) 10:307-17. doi: 10.1038/mi.2016.128

21. Strober W, Fuss IJ, Blumberg RS. The immunology of mucosal models of inflammation. Annu Rev Immunol. (2002) 20:495549. doi: 10.1146/annurev.immunol.20.100301.064816

22. Rolig AS, Mittge EK, Ganz J, Troll JV, Melancon E, Wiles TJ, et al. The enteric nervous system promotes intestinal health by constraining microbiota composition. PLoS Biol. (2017) 15:1-22. doi: 10.1371/journal.pbio.2000689 
23. Ding L, Lu Z, Foreman O, Tatum R, Lu Q, Renegar R, et al. Inflammation and disruption of the mucosal architecture in claudin-7-deficient mice. Gastroenterology. (2012) 142:305-15. doi: 10.1053/j.gastro.2011.10.025

24. Clayburgh DR, Barrett TA, Tang Y, Meddings JB, Van Eldik LJ, Watterson DM, et al. Epithelial myosin light chain kinase-dependent barrier dysfunction mediates $\mathrm{T}$ cell activation-induced diarrhea in vivo. J Clin Invest. (2005) 115:2702-15. doi: 10.1172/JCI24970

25. Zeissig S, Bürgel N, Günzel D, Richter J, Mankertz J, Wahnschaffe U, et al. Changes in expression and distribution of claudin 2, 5 and 8 lead to discontinuous tight junctions and barrier dysfunction in active Crohn's disease. Gut. (2007) 56:61-72. doi: 10.1136/gut.2006.094375

26. Gu M, Jia Q, Zhang Z, Bai $N$, Xu X, Xu B. Soya-saponins induce intestinal inflammation and barrier dysfunction in juvenile turbot (Scophthalmus maximus). Fish Shellfish Immunol. (2018) 77:264-72. doi: 10.1016/j.fsi.2018.04.004

27. Zhao Y, Qin G, Sun Z, Che D, Bao N, Zhang X. Effects of soybean agglutinin on intestinal barrier permeability and tight junction protein expression in weaned piglets. Int J Mol Sci. (2011) 12:8502-12. doi: 10.3390/ijms12128502

28. Okayasu I, Hatakeyama S, Yamada M, Ohkusa T, Inagaki Y, Nakaya R. A novel method in the induction of reliable experimental acute and chronic ulcerative colitis in mice. Gastroenterology. (1990) 98:694702. doi: 10.1016/0016-5085(90)90290-H

29. Kiesler P, Fuss IJ, Strober W. Experimental models of inflammatory bowel diseases. Med Hyg. (2001) 59:241-8. doi: 10.1016/j.jcmgh.2015.01.006

30. Hermiston ML, Gordon JI. In vivo analysis of cadherin function in the mouse intestinal epithelium: essential roles in adhesion, maintenance of differentiation, and regulation of programmed cell death. J Cell Biol. (1995) 129:489-506. doi: 10.1083/jcb.129.2.489

31. Van der Sluis M, De Koning BAE, De Bruijn ACJM, Velcich A, Meijerink JPP, Van Goudoever JB, et al. Muc2-deficient mice spontaneously develop colitis, indicating that MUC2 is critical for colonic protection. Gastroenterology. (2006) 131:117-29. doi: 10.1053/j.gastro.2006.04.020

32. Nishida A, Lau CW, Zhang $M$, Andoh A, Shi HN, Mizoguchi $\mathrm{E}$, et al. The membrane-bound mucin $\mathrm{Mucl}$ regulates $\mathrm{T}$ helper 17-cell responses and colitis in mice. Gastroenterology. (2012) 142:865-74.e2. doi: 10.1053/j.gastro.2011.12.036

33. Panwala CM, Jones JC, Viney JL. A novel model of inflammatory bowel disease: mice deficient for the multiple drug resistance gene, mdr1a, spontaneously develop colitis. J Immunol. (1998) 161:5733-44.

34. Kobayashi M, Kweon MN, Kuwata H, Schreiber RD, Kiyono H, Takeda K, et al. Toll-like receptor-dependent production of IL-12p40 causes chronic enterocolitis in myeloid cell-specific Stat3-deficient mice. J Clin Invest. (2003) 111:1297-308. doi: 10.1172/JCI17085

35. Sellon RK, Tonkonogy S, Schultz M, Dieleman LA, Grenther W, Balish E, et al. Resident enteric bacteria are necessary for development of spontaneous colitis and immune system activation in interleukin-10-deficient mice. Infect Immun. (1998) 66:5224-31. doi: 10.1128/IAI.66.11.5224-523 1.1998

36. Rath HC, Schultz M, Freitag R, Dieleman LA, Li F, Linde HJ, et al. Different subsets of enteric bacteria induce and perpetuate experimental colitis in rats and mice. Infect Immun. (2001) 69:2277-85. doi: 10.1128/IAI.69.4.2277-2285.2001

37. Baümler AJ, Sperandio V. Interactions between the microbiota and pathogenic bacteria in the gut. Nature. (2016) 535:8593. doi: 10.1038/nature18849

38. Rolig AS, Parthasarathy R, Burns AR, Bohannan BJM, Guillemin K. Individual members of the microbiota disproportionately modulate host innate immune responses. Cell Host Microbe. (2015) 18:613-20. doi: 10.1016/j.chom.2015.10.009

39. Navarrete P, Fuentes P, De la Fuente L, Barros L, Magne F, Opazo R, et al. Short-term effect of dietary soybean meal and lactic acid bacteria on the intestinal morphology and microbiota of Atlantic salmon (Salmo salar). Aquac Nutr. (2013) 19:827-36. doi: 10.1111/anu.12047

40. Navarrete P, Espejo RT, Romero J. Molecular analysis of microbiota along the digestive tract of juvenile atlantic salmon (Salmo salar L.). Microb Ecol. (2009) 57:550-61. doi: 10.1007/s00248-008-9448-x

41. Demir AK, Demirtas A, Kaya SU, Tastan I, Butun I, Sagcan M, et al. The relationship between the neutrophil-lymphocyte ratio and disease activity in patients with ulcerative colitis. Kaohsiung J Med Sci. (2015) 31:58590. doi: 10.1016/j.kjms.2015.10.001

42. Kühl AA, Kakirman H, Janotta M, Dreher S, Cremer P, Pawlowski NN, et al. Aggravation of different types of experimental colitis by depletion or adhesion blockade of neutrophils. Gastroenterology. (2007) 133:188292. doi: 10.1053/j.gastro.2007.08.073

43. Nemoto Y, Kanai T, Tohda S, Totsuka T, Okamoto R, Tsuchiya $\mathrm{K}$, et al. Negative feedback regulation of colitogenic CD4+ $\mathrm{T}$ cells by increased granulopoiesis. Inflamm Bowel Dis. (2008) 14:1491-503. doi: 10.1002/ibd.20531

44. Stagaman K, Cepon-Robins TJ, Liebert MA, Gildner TE, Urlacher SS, Madimenos FC, et al. Market integration predicts human gut microbiome attributes across a gradient of economic development. mSystems. (2018) 3:e00122-17. doi: 10.1128/mSystems.00122-17

45. Kucharzik T, Walsh S V, Chen J, Parkos CA, Nusrat A. Neutrophil transmigration in inflammatory bowel disease is associated with differential expression of epithelial intercellular junction proteins. Am J Pathol. (2001) 159:2001-9. doi: 10.1016/S0002-9440(10)63051-9

46. Westerfield M. The Zebrafish Book. A Guide for the Laboratory Use of Zebrafish (Danio rerio). 3rd ed. Eugene, OR: University of Oregon Press (1995).

47. Cocchiaro JL, Rawls JF. Microgavage of zebrafish larvae. J Vis Exp. (2013) 20:e4434. doi: 10.3791/4434

48. Neal JT, Peterson TS, Kent ML, Guillemin K. H. pylori virulence factor CagA increases intestinal cell proliferation by Wnt pathway activation in a transgenic zebrafish model. Dis Model Mech. (2013) 6:80210. doi: $10.1242 / \mathrm{dmm} .011163$

49. Bates JM, Mittge E, Kuhlman J, Baden KN, Cheesman SE, Guillemin K. Distinct signals from the microbiota promote different aspects of zebrafish gut differentiation. Dev Biol. (2006) 297:374-86. doi: 10.1016/j.ydbio.2006.05.006

50. Pham LN, Kanther M, Semova I, Rawls JF. Methods for generating and colonizing gnotobiotic zebrafish. Nat Protoc. (2008) 3:1862-75. doi: 10.1038/nprot.2008.186

51. Romero J, Navarrete P. 16S rDNA-based analysis of dominant bacterial populations associated with early life stages of coho salmon (Oncorhynchus kisutch). Microb Ecol. (2006) 51:422-30. doi: 10.1007/s00248-0069037-9

52. Caporaso JG, Lauber CL, Walters WA, Berg-Lyons D, Lozupone CA, Turnbaugh PJ, et al. Global patterns of 16S rRNA diversity at a depth of millions of sequences per sample. Proc Natl Acad Sci USA. (2011) 108:451622. doi: $10.1073 /$ pnas. 1000080107

53. Caporaso JG, Fierer N, Peña AG, Goodrich JK, Gordon JI, Huttley GA, et al. QIIME allows analysis of high-throughput community sequencing data. Nat Methods. (2010) 7:335-6. doi: 10.1038/nmeth.f.303

54. Haas BJ, Gevers D, Earl AM, Feldgarden M, Ward DV, Giannoukos G, et al. Chimeric 16S rRNA sequence formation and detection in sanger and 454-pyrosequenced PCR amplicons. Genome Res. (2011) 21:494504. doi: 10.1101/gr.112730.110

55. Bokulich NA, Subramanian S, Faith JJ, Gevers D, Gordon JI, Knight R, et al. Quality-filtering vastly improves diversity estimates from illumina amplicon sequencing. Nat Methods. (2013) 10:57-59. doi: 10.1038/nmeth.2276

56. Segata N, Izard J, Waldron L, Gevers D, Miropolsky L, Garrett WS, et al. Metagenomic biomarker discovery and explanation. Genome Biol. (2011) 12:R60. doi: 10.1186/gb-2011-12-6-r60

57. Callahan BJ, McMurdie PJ, Rosen MJ, Han AW, Johnson AJA, Holmes SP. DADA2: High-resolution sample inference from Illumina amplicon data. Nat Methods. (2016) 13:581-3. doi: 10.1038/nmeth.3869

Conflict of Interest: The authors declare that the research was conducted in the absence of any commercial or financial relationships that could be construed as a potential conflict of interest.

Copyright (C) 2020 Solis, Hamilton, Caruffo, Garcia-Lopez, Navarrete, Guillemin and Feijoo. This is an open-access article distributed under the terms of the Creative Commons Attribution License (CC BY). The use, distribution or reproduction in other forums is permitted, provided the original author(s) and the copyright owner(s) are credited and that the original publication in this journal is cited, in accordance with accepted academic practice. No use, distribution or reproduction is permitted which does not comply with these terms. 